\title{
New Controllability Results of Fractional Nonlocal Semilinear Evolution Systems with Finite Delay
}

\author{
Daliang Zhao ${ }^{1}{ }^{1}$ and Juan Mao (D) $^{2}$ \\ ${ }^{1}$ School of Mathematics and Statistics, Shandong Normal University, Jinan 250014, China \\ ${ }^{2}$ Department of Basic Courses, Shandong Polytechnic, Jinan 250104, China \\ Correspondence should be addressed to Daliang Zhao; dlzhao928@sdnu.edu.cn
}

Received 9 May 2020; Accepted 19 June 2020; Published 13 July 2020

Academic Editor: Quanxin Zhu

Copyright ( 2020 Daliang Zhao and Juan Mao. This is an open access article distributed under the Creative Commons Attribution License, which permits unrestricted use, distribution, and reproduction in any medium, provided the original work is properly cited.

\begin{abstract}
In the present paper, sufficient conditions ensuring the complete controllability for a class of semilinear fractional nonlocal evolution systems with finite delay in Banach spaces are derived. The new results are obtained under a weaker definition of complete controllability we introduced, and then the Lipschitz continuity and other growth conditions for the nonlinearity and nonlocal item are not required in comparison with the existing literatures. In addition, an appropriate complete space and a corresponding time delay item are introduced to conquer the difficulties caused by time delay. Our main tools are properties of resolvent operators, theory of measure of noncompactness, and Mönch fixed point theorem.
\end{abstract}

\section{Introduction}

In recent decades, various research studies on fractional differential systems are growing vigorously, which are chiefly due to the enormous scope and extensive applications of fractional calculus theory in mathematics, biology, physics, economics, and engineering science [1-9].

As we all know, time delay effects exist widely in various fields such as communication security, weather predicting, and population dynamics. The difficulties in the study of these fields lie in the time lag effect for the systems caused by the delay. One can see [10-20], for further details. It should be noted that, in the case of research on fractional delay evolution systems, it is still in the initial stage. On the contrary, fractional calculus has also been applied to controllability issues in recent years. As one of the most important notions in control theory of mathematics, controllability has significant influence on the fields of control and engineering. We point out that complete controllability of infinite dimensional systems with noncompact semigroup is an important research direction, and there have been many outstanding achievements in this regard such as [21-26]. For more details of other research results on control theory, please refer to [27-40] and the reference therein.

As we have seen, there is scarcely any results on complete controllability of fractional nonlocal evolution equations with delay in Banach spaces, except [21, 23, 24]. However, the Lipschitz and certain growth conditions on nonlocal item and nonlinearity are still necessary for [21]. In [23], the authors supposed the nonlinear function and nonlocal item to be Lipschitz continuous and the semigroup generated by the considered system to be compact. In [24], nonlinear function was imposed some growth conditions, and the resolvent operator used to define the mild solution was compact. However, it is a little pity that these suppositions are usually difficult to be made to work in numerous specific applications. In fact, many excellent achievements concerning complete controllability of various nonlinear differential systems, such as those in Debbouche and Baleanu, [11], Nirmala et al. [22], and Wang and Zhou [25], have been derived during recent years. However, the limitation also lies in that the nonlinear functions here are all provided with the Lipschitz continuity or other growth assumptions. In 
addition, the compactness or other assumptions of $C_{0}$ semigroup actually prevents us from studying complete controllability in the infinite dimensional space.

The current paper, inspired by the aforementioned analyses, is to address the complete controllability of the following fractional nonlocal semilinear evolution equations with finite delay in Banach spaces:

$$
\begin{cases}D^{q} u(t)=A u(t)+f\left(t, u(t), u_{t}\right)+B \mu(t), & \text { a.e. } t \in I:=[0, T], \\ u(t)+M(t, u)=\phi(t), & t \in[-r, 0],\end{cases}
$$

where $D^{q}$ denotes the Caputo derivative of order $q \in(0,1)$. $u: I \longrightarrow X, \mu \in L^{2}(I, U)$, and $B: U \longrightarrow X$ is a bounded linear operator, where $X$ and $U$ are Banach spaces. $A: \mathscr{D} \subset X \longrightarrow X$ is a closed linear unbounded operator on $X$ with dense domain $\mathscr{D} . \phi(t) \in C([-r, 0], X) . f$ and $M$ are given functions satisfying certain appropriate conditions that will be given later.

The proposed fractional evolution system (1) here, which generalizes the case of integral (first) order differential equations studied in [41] about the complete controllability, has more extensive and valid applications in contrast to the abovementioned literatures [11,21-25] as follows. First of all, under the new concept of complete controllability we drew into, that is, a weaker definition of complete controllability than the existing notion, the nonlinear function and nonlocal item here are only allowed to be continuous instead of other restrictions such as Lipschitz continuity and certain growth conditions. Secondly, compactness of the $C_{0}$ semigroup and the resolvent operator used to define the mild solution for system (1) is no longer needed. Last but not the least, obstacles in the estimation of Kuratowski measures of noncompactness caused by time delay have been conquered in a new complete space with corresponding defined delay function.

An outline of the current paper is as follows. Some essential preparations are made in Section 2. In Section 3, we derive a sufficient condition on complete controllability for the addressed systems. In Section 4, we give an example to illustrate the obtained new results.

\section{Preliminaries}

Let $C(I, X)$ be the space of continuous functions from $I$ into $X$ provided with the supreme norm $\|\cdot\|_{C(I, X)}$. Similarly, $C([-r, T], X)$ denotes the Banach space of continuous functions from $[-r, T]$ to $X$ with the usual supreme norm. If $T=0$, we denote the norm of this space simply by $\|\cdot\|_{r}$. The domain $\mathscr{D}$ is endowed with the graph norm $\|u\|_{\mathscr{D}}=$ $\|u\|+\|A u\|$, where $\|\cdot\|$ denotes the norm of Banach space $X$. For $q \in(0,1), C^{q}(I, X)$ stands for the space of all the continuous functions from $I$ into $X$ equipped with the norm $\|u\|_{C^{q}(I, X)}=\|u\|_{C_{(I, X)}}+[|u|]_{C^{q}(I, X)}$, where $\quad[|u|]_{C^{q}(I, X)}=$ $\sup _{t, s \in I, t \neq s}\|u(t)-u(s)\| /(t-s)^{q}$. Throughout this paper, $\mathscr{L}(X, Y)$ denotes the space of bounded linear operators from $X$ into Banach space $Y$ provided with the operator norm $\|\cdot\|_{\mathscr{L}(X, Y)}$.
In order to conquer the inconveniences caused by time delay in the study of complete controllability, for each $u \in C(I, X), t \in I$, and the function $\phi(t)$ in (1), we draw into the function $u_{t}$ defined by

$$
u_{t}(\theta)= \begin{cases}u(t+\theta), & t+\theta \geq 0, \\ \phi(t+\theta), & t+\theta \leq 0,\end{cases}
$$

for each $\theta \in[-r, 0]$. Simple check indicates $x_{t} \in$ $L([-r, 0], X)$, where $L([-r, 0], X)$ stands for a complete integrable space consists of integrable functions from $[-r, 0]$ into $X$.

Remark 1. Under the function $u_{t}$ defined in (2) in complete space $L([-r, 0], X)$, we can get over the difficulties caused by time delay in the process of estimating Kuratowski measures of noncompactness (for details, please see Lemmas 4 and 5).

Next, we list the well-known definitions as follows.

Definition 1 (see [5]). The fractional integral with order $q>0$ for a function $u:(0,+\infty) \longrightarrow R$ can be defined as

$$
I_{0^{+}}^{q} u(t)=\frac{1}{\Gamma(q)} \int_{0}^{t}(t-s)^{q-1} u(s) \mathrm{d} s,
$$

provided that the right-side integral is pointwise defined on $[0,+\infty)$.

Definition 2 (see [5]). The Caputo fractional derivative with order $q>0$ for a function $u:(0,+\infty) \longrightarrow R$ is written as

$$
D_{0^{+}}^{q} u(t)=\frac{1}{\Gamma(n-q)} \int_{0}^{t} \frac{u^{(n)}(s)}{(t-s)^{q-n+1}} \mathrm{~d} s,
$$

where $n=[q]+1$, provided the right-side integral is pointwise defined on $[0,+\infty)$.

Definition 3 (see [42]). The bounded linear operator $\{\mathfrak{I}(t)\}_{t \geq 0} \subset \mathscr{L}(X)$ on $X$ is defined as a resolvent operator of the following integral equation:

$$
u(t)=\int_{0}^{t} \sigma(t-s) A u(s) \mathrm{d} s, \quad t \geq 0,
$$

where scalar kernel $\sigma \in L_{l o c}^{1}\left(R^{+}\right)$and $\sigma \equiv 0$, provided that it satisfies

(i) $\mathfrak{I}(t)$ is strongly continuous, $\mathfrak{I}(0)=I$

(ii) $\mathfrak{I}(t)$ commutes with $A$, that is, $\mathfrak{I}(t) \mathscr{D} \subset \mathscr{D}$, and $A \mathfrak{I}(t) u=\mathfrak{I}(t) A u$ for each $u \in \mathscr{D}$ and each $t \geq 0$

(iii) $\mathfrak{I}(t) u=u+\int_{0}^{t} \sigma(t-s) A \mathfrak{I}(s) u \mathrm{~d} s$, for all $u \in \mathscr{D}$, $t \geq 0$

Definition 4 (see [42]). Suppose $\mathfrak{\Im}(\cdot) u \in W_{\text {loc }}^{1,1}\left(R^{+}, X\right)$ for each $u \in \mathscr{D}$, and there exists a function $\varphi \in L_{l o c}^{1}\left(R^{+}\right)$, which satisfy

$$
\|\dot{\mathfrak{J}}(t) u\| \leq \varphi(t)\|u\|_{\mathscr{D}}, \quad \text { a.e. on } R^{+}, \forall u \in \mathscr{D} .
$$

Then, we call $\mathfrak{\Im}(t)$ a differentiable resolvent operator of (5). 
Next, we focus on the following equation:

$$
u(t)=g(t)+\int_{0}^{t} \sigma(t-s) A u(s) \mathrm{d} s, \quad t \in I,
$$

where $g \in L^{1}(I, X)$, and list the definition of mild solution of (7) as below on the basis of literature [42].

Definition 5. We call $u \in C(I, X)$ a mild solution for (7) if $\int_{0}^{t} \sigma(t-s) u(s) \mathrm{d} s \in \mathscr{D}$, which satisfies

$$
u(t)=g(t)+A \int_{0}^{t} \sigma(t-s) u(s) \mathrm{d} s,
$$

for each $t \in I$.

Taking advantage of properties of the differentiable resolvent operator, we present the equivalent definition of mild solution for (7).

Lemma 1 (see [42]). Assume that $\mathfrak{\Im}(t)$ is a differentiable resolvent operator for (7) and $g \in C(I, \mathscr{D})$. Then,

$$
u(t)=\int_{0}^{t} \dot{\mathfrak{J}}(t-s) g(s) \mathrm{d} s+g(t), \quad t \in I,
$$

is a mild solution of (7).

Before going further, we now recall below a few relevant properties of Kuratowski measures of noncompactness, which will play a critical role in our next proof of complete controllability. For further details, please see [43].

Lemma 2. Let $X$ be a Banach space and $\gamma(\cdot)$ be the Kuratowski measures of noncompactness which is given by $\gamma(\Omega)=$ $\inf \left\{\delta>0: \Omega=\cup_{i=1}^{k} \Omega_{i}\right.$ with $\left.\operatorname{diam}\left(\Omega_{i}\right) \leq \delta, \quad i=1,2, \ldots, k\right\}$ for a bounded subset $\Omega$ in $X$.

(I) Let $D_{1}, D_{2}$ be bounded sets of $X$ and $\lambda \in R$. Then,

(i) $\gamma\left(D_{1}\right)=0 \Longleftrightarrow D_{1}$ is relatively compact

(ii) $\gamma\left(\lambda D_{1}\right)=|\lambda| \gamma\left(D_{1}\right)$

(iii) $\gamma\left(D_{1}+D_{2}\right) \leq \gamma\left(D_{1}\right)+\gamma\left(D_{2}\right)$

(II) Assume that $D=\left\{u_{n}\right\}$ is a countable set of strongly measurable functions from I into Banach space $X$, and there exists a function $\psi \in L^{1}(I)$ such that $\left\|u_{n}(t)\right\| \leq \psi(t)$, a.e. $t \in I, n=1,2, \ldots$, then $\gamma(D(t))$ is integrable on $I$, which satisfies

$$
\gamma\left(\left\{\int_{I} u_{n}(t) \mathrm{d} t: n \in N\right\}\right) \leq 2 \int_{I} \gamma(D(t)) \mathrm{d} t .
$$

For the convenience of expression, the Kuratowski measures of noncompactness of a bounded subset in spaces
$X, C(I, X)$ and $L([-r, 0], X)$ are all written as $\gamma(\cdot)$, on the premise of no confusion.

Lemma 3 (Mönch). If there is a closed and convex set $D \subset X$, where $X$ is a Banach space, $u_{0} \in D$, and suppose that $A: D \longrightarrow D$ is continuous and satisfies $C \subset D$ countable, $C \subset \overline{c o}\left(\left\{u_{0}\right\} \cup A(C)\right) \Longrightarrow C$ is relatively compact. Then, $A$ has a fixed point in $D$.

To end this section, we give the following useful lemmas.

Lemma 4. Assume that $u_{n}$ converges to $u_{0}$ in $C(I, X)$ as $n \longrightarrow+\infty$. Then, $\left(u_{n}\right)_{t}$ converges to $\left(u_{0}\right)_{t}$ in $L([-r, 0], X)$ for every $t \in I$ as $n \longrightarrow+\infty$.

Proof. Based on the definition in (2), we know

$$
\left\|\left(u_{n}\right)_{t}-\left(u_{0}\right)_{t}\right\|_{L[-r, 0]}= \begin{cases}\int_{0}^{t}\left\|u_{n}(s)-u_{0}(s)\right\| d s, & t \leq r, \\ \int_{t-r}^{t}\left\|u_{n}(s)-u_{0}(s)\right\| d s, & t \geq r,\end{cases}
$$

which implies that

$$
\left\|\left(u_{n}\right)_{t}-\left(u_{0}\right)_{t}\right\|_{L[-r, 0]} \leq r\left\|u_{n}-u_{0}\right\|_{C(I, X)}, \quad \forall t \in I .
$$

This completes the proof.

From the definition of Kuratowski measures of noncompactness in Lemma 2, we can infer the following lemma.

Lemma 5. Let $D=\left\{u_{n}\right\}_{n=1}^{\infty}$ be any bounded countable sequence in $C(I, X)$. Then, for each $t \in I$, one has

$$
\gamma\left(D_{t}\right) \leq r \gamma(D)
$$

where $D_{t}=\left\{\left(u_{n}\right)_{t}\right\}_{n=1}^{\infty}$.

\section{Main Results}

In the sequel, we assume that $A$ admits a differentiable resolvent operator $\{\mathfrak{\Im}(t)\}_{t \geq 0}$ on $X$. Based on Definition 5 and the Riemann-Liouville standard fractional integral, the mild solution of system (1) can be defined as follows.

Definition 6. For each $\mu \in L^{2}(I, U)$, a function $u \in C(J, X)$ is said to be a mild solution of the considered system (1) on $J$, if $\int_{0}^{t}(t-s)^{q^{-1}} u(s) \mathrm{d} s \in \mathscr{D}$ for any $t \in\left[0, T^{*}\right]$ and

$$
u(t)= \begin{cases}\phi(0)-M(0, u)+\frac{1}{\Gamma(q)} A \int_{0}^{t} \frac{u(s)}{(t-s)^{1-q}} d s+\frac{1}{\Gamma(q)} \int_{0}^{t} \frac{f\left(s, u(s), u_{s}\right)+B \mu(s)}{(t-s)^{1-q}} \mathrm{~d} s, & t \in\left[0, T^{*}\right], \\ \phi(t)-M(t, u), & t \in[-r, 0]\end{cases}
$$

where $J=\left[-r, T^{*}\right], T^{*} \in(0, T]$. 
Definition 7 (complete controllability). We shall call system (1) is completely controllable on $I=[0, T]$, if for each initial function $\phi(t) \in C([-r, 0], X)$ and $u_{1} \in X$, there exist a corresponding control $\mu \in L^{2}(I, U)$ and a real number $T^{*} \in(0, T]$ such that the mild solution $u$ of (1) on $J=\left[-r, T^{*}\right]$ satisfies $u\left(T^{*}\right)=u_{1}$.

Remark 2. In comparison with the existing notion in $[11,21,22,25,41]$, in which $T^{*}$ is equal to $T$, our concept in which $T^{*} \in(0, T]$ is weaker can be regarded as an extension of the present notion of complete controllability.

Before presenting and proving the main results, we firstly list the hypotheses as below:

(H1) $f \in C(I \times X \times L([-r, 0], X), \mathscr{D}), \quad \phi(0) \in \mathscr{D}$ and satisfies

(i) $f$ maps bounded sets in $I \times X \times L([-r, 0], X)$ into bounded sets in $\mathscr{D}$.

(ii) There exist a constant $q_{1} \in(0, q)$ and a function $m \in L^{\left(1 / q_{1}\right)}\left(I, R^{+}\right)$such that, for any bounded subsets $D_{1} \subset X, D_{2} \subset L([-r, 0], X)$ $\gamma\left(f\left(t, D_{1}, D_{2}\right)\right) \leq m(t)\left(\gamma\left(D_{1}\right)+\gamma\left(D_{2}\right)\right), \quad t \in I$.

(H2) (i) The linear operator $B: L^{2}(I, U) \longrightarrow L^{1}(I, \mathscr{D})$ is bounded, and there exists a constant $N_{1}>0$ satisfying $\|B\|_{\mathscr{L}(U, \mathscr{D})} \leq N_{1}$.

(ii) Linear operators $\mathfrak{R}(t), t \in I$, denoted by $\mathfrak{R}(\cdot)$ from $L^{2}(I, U)$ to $X$ are defined as

$\mathfrak{R}(t) \mu=\Pi_{B}(t, \mu)+\int_{0}^{t} \dot{\mathfrak{I}}(t-s) \Pi_{B}(s, \mu) \mathrm{d} s, \quad t \in I$,

where $\Pi_{B}(t, \mu)=1 / \Gamma(q) \int_{0}^{t}(t-s)^{q-1} B \mu(s) \mathrm{d} s$ has invertible operators $\Re^{-1}(\cdot)$ taking values in $L^{2}(I, U) / \operatorname{ker} \Re(\cdot)$, which satisfy, for some constant $N_{2}>0, \quad \sup \left\|\Re^{-1}(\cdot)\right\|_{\mathscr{L}\left(X, L^{2}(I, U) / \operatorname{ker} \Re(\cdot)\right)} \leq N_{2}$, and there is a constant $q_{2} \in(0, q)$ and a function $p \in L^{\left(1 / q_{2}\right)}\left(I, R^{+}\right)$satisfying

$$
\gamma\left(\Re^{-1}(\cdot)(D)(s)\right) \leq p(s) \gamma(D), \quad s \in I,
$$

for any bounded subset $D \subset X$.

(H3) (i) For every $t \in[-r, 0], \quad M(t, \cdot): C([-r, T]$, $X) \longrightarrow X$ is a strict $k$-set contraction and for every $u \in C([-r, T], X), M(t, u)$ is continuous with respect to each $t \in[-r, 0]$.

(ii) $M(0, \cdot): C([-r, T], X) \longrightarrow \mathscr{D}$ and there exists a positive constant $G$ such that $\|M(0, u)\|_{\mathscr{D}} \leq G$ for every $u \in C([-r, T], X)$.

(H4) The following estimation is valid:

$$
k M\left(1+2\|\varphi\|_{L^{1}(I)}\right)<1,
$$

where

$$
\begin{aligned}
& M=1+\frac{2 N_{1} L\left(1+2\|\varphi\|_{L^{1}(I)}\right)}{\Gamma(q)}, \\
& L=T^{q-q_{2}}\left(\frac{1-q_{2}}{q-q_{2}}\right)^{1-q_{2}}\|p\|_{L^{\left(1 / q_{2}\right)}},
\end{aligned}
$$

and $\varphi$ is the function mentioned in Definition 4 .

In the following, let $R_{0}$ be a fixed constant satisfying $R_{0}>\left(\|\phi(0)\|_{\mathscr{D}}+G\right)\left(1+\|\varphi\|_{L^{1}(I)}\right):=N_{0}$. From (H1), take

$$
\begin{aligned}
M_{0} & =\sup \left\{\|f(t, u, v)\|_{\mathscr{D}}:\|u\|_{C(I, X)} \leq R_{0},\|v\|_{L[-r, 0]}\right. \\
& \left.\leq r\left(\|\phi\|_{r}+R_{0}\right), \quad t \in I\right\} .
\end{aligned}
$$

For simplicity, let

$$
\begin{aligned}
\Pi(t, u, \mu)= & \frac{1}{\Gamma(q)} \int_{0}^{t}(t-s)^{q-1} f\left(s, u(s), u_{s}\right) \mathrm{d} s \\
& +\frac{1}{\Gamma(q)} \int_{0}^{t}(t-s)^{q-1} B \mu(s) \mathrm{d} s,
\end{aligned}
$$

and set

$$
\lambda=\frac{2(r+1)}{\Gamma(q)}\left(\frac{1-q_{1}}{q-q_{1}}\right)^{1-q_{1}}\|m\|_{L\left(1 / q_{1}\right)} .
$$

By means of conditions (H2) and (H4), for any $u(\cdot) \in C(I, X)$ and any $u_{1} \in X, t \in I$, define a feedback control function

$$
\begin{aligned}
\mu_{u}(t):= & \Re^{-1}\left(T^{*}\right)\left(u_{1}-\phi(0)+M(0, u)-\Pi_{f}\left(T^{*}, u\right)\right. \\
& \left.-\int_{0}^{T^{*}} \dot{\mathfrak{\Im}}\left(T^{*}-s\right)\left(\phi(0)-M(0, u)+\Pi_{f}(s, u)\right) \mathrm{d} s\right)(t),
\end{aligned}
$$

where

$$
T^{*}=\min \left\{T,\left(\frac{\left(R_{0}-N_{0}\right) \Gamma(q+1)}{\left(M_{0}+N_{1} M_{1}\right)\left(1+\|\varphi\|_{L^{1}(l)}\right)}\right)^{(1 / q)},\left(\frac{1-k M\left(1+2\|\varphi\|_{L^{1}(l)}\right)}{\lambda M\left(1+2\|\varphi\|_{L^{(}(l)}\right)}\right)^{(1 / q)}\right\},
$$

$$
M_{1}=N_{2}\left(\left(1+\|\varphi\|_{L^{1}(I)}\right)\left(\|\phi(0)\|_{\mathscr{D}}+G+\frac{M_{0} T^{q}}{\Gamma(q+1)}\right)+\left\|u_{1}\right\|\right),
$$

$$
\Pi_{f}(t, u)=\frac{1}{\Gamma(q)} \int_{0}^{t}(t-s)^{q-1} f\left(s, u(s), u_{s}\right) \mathrm{d} s .
$$

\section{Denote}

$$
\begin{aligned}
\Omega= & \left\{u \in C(J, X):\|u\|_{C\left(\left[0, T^{*}\right], X\right)} \leq R_{0}, \sup _{t \in\left[0, T^{*}\right]}\left\|u_{t}\right\|_{L[-r, 0]}\right. \\
& \left.\leq r\left(\|\phi\|_{r}+R_{0} 0\right) ; u(t)=\phi(t)-M(t, u), t \in[-r, 0],\right\},
\end{aligned}
$$


then $\Omega$ is clearly a closed convex set in $C(J, X)$. From Lemma 1 , we thus define an operator $\Psi: C(J, X) \longrightarrow C(J, X)$ which is given by

$$
(\Psi u)(t)= \begin{cases}\phi(0)-M(0, u)+\Pi\left(t, u, \mu_{u}\right)+\int_{0}^{t} \dot{\mathfrak{J}}(t-s)\left(\phi(0)-M(0, u)+\Pi\left(s, u, \mu_{u}\right)\right) \mathrm{d} s, & t \in\left[0, T^{*}\right], \\ \phi(t)-M(t, u), & t \in[-r, 0] .\end{cases}
$$

For the purpose of simplifying our next proof processes, we give the following conclusions.

$$
[|\Pi(\cdot, u, \mu)|]_{C^{q}} \leq \frac{2}{q}\left(\|f(\cdot, u(\cdot), u .)\|_{C(I, X)}+\|B\|_{\mathscr{L}(U, X)}\|\mu(\cdot)\|_{L^{2}}\right) .
$$

Lemma 6. Assume $f(\cdot, u(\cdot), u.) \in C(I \times X \times L([-r, 0]$, $X), X)$ and $\mu(\cdot) \in L^{2}(I, U)$. Then, $\Pi(\cdot, u, \mu) \in C^{q}(I, X)$, and

$$
\begin{aligned}
\|\Pi(t+h, u, \mu)-\Pi(t, u, \mu)\| \\
\leq \int_{0}^{t}\left((t-s)^{q-1}-(t+h-s)^{q-1}\right)\left(\left\|f\left(s, u(s), u_{s}\right)\right\|+\|B \mu(s)\|\right) \mathrm{d} s \\
\quad+\int_{t}^{t+h}(t+h-s)^{q-1}\left(\left\|f\left(s, u(s), u_{s}\right)\right\|+\|B \mu(s)\|\right) \mathrm{d} s \\
\leq\left(\frac{t^{q}-(t+h)^{q}+h^{q}}{q}+\frac{h^{q}}{q}\right)\left(\|f(\cdot, u(\cdot), u \cdot)\|_{C(I, X)}+\|B\|_{\mathscr{L}(U, X)}\|\mu(\cdot)\|_{L^{2}}\right) \\
\leq \frac{2 h^{q}}{q}\left(\|f(\cdot, u(\cdot), u .)\|_{C(I, X)}+\|B\|_{\mathscr{L}(U, X)}\|\mu(\cdot)\|_{L^{2}}\right),
\end{aligned}
$$

which implies that $[|\Pi(\cdot, u, \mu)|]_{C^{q}} \leq(2 / q)(\| f(\cdot, u(\cdot)$, $u$. $\left.\left\|_{C(I, X)}+\right\| B\left\|_{\mathscr{L}(U, X)}\right\| \mu(\cdot) \|_{L^{2}}\right)$ and $\Pi(\cdot, u, \mu) \in C^{q}(I, X)$. The conclusion follows.
Lemma 7. Assume that conditions (H1) (i) and (H2)-(H4) hold. Then, the operator $\Psi: \Omega \longrightarrow \Omega$ is equicontinuous on $J$.

Proof. Firstly, we prove that $\Psi(\Omega) \subseteq \Omega$. In view of assumptions (H1) (i), (H2), and (H3) (ii), we can derive

$$
\begin{aligned}
\left\|\mu_{u}(t)\right\| \leq & N_{2}\left(\left\|u_{1}\right\|+\|\phi(0)\|_{\mathscr{D}}+G+\int_{0}^{T^{*}} \varphi\left(T^{*}-s\right)\left(\|\phi(0)\|_{\mathscr{D}}+G\right) \mathrm{d} s\right) \\
& +\frac{N_{2}}{\Gamma(q)}\left(\int_{0}^{T^{*}}\left(T^{*}-s\right)^{q-1}\left\|f\left(s, u(s), u_{s}\right)\right\|_{\mathscr{D}} \mathrm{d} s+\int_{0}^{T^{*}} \varphi\left(T^{*}-s\right)\left(\int_{0}^{s}(s-\eta)^{q-1}\left\|f\left(\eta, u(\eta), u_{\eta}\right)\right\|_{\mathscr{D}} d \eta\right) \mathrm{d} s\right) \\
& \leq N_{2}\left(\left\|u_{1}\right\|+\|\phi(0)\|_{\mathscr{D}}+G+\|\varphi\|_{L^{1}(I)}\left(\|\phi(0)\|_{\mathscr{D}}+G\right)\right)+N_{2}\left(\frac{M_{0} T^{q}}{\Gamma(q+1)}+\|\varphi\|_{L^{1}(I)} \frac{M_{0} T^{q}}{\Gamma(q+1)}\right) \\
& \leq N_{2}\left(\left(\|\varphi\|_{L^{1}(I)}+1\right)\left(\|\phi(0)\|_{\mathscr{D}}+G+\frac{M_{0} T^{q}}{\Gamma(q+1)}\right)+\left\|u_{1}\right\|\right)=M_{1}, \quad t \in I .
\end{aligned}
$$


Observing (H2), for any $u \in \Omega$ and $t \in\left[0, T^{*}\right]$, we deduce

$$
\begin{aligned}
\left\|\Pi\left(t, u, \mu_{u}\right)\right\|_{\mathscr{D}} \leq & \frac{1}{\Gamma(q)} \int_{0}^{t}(t-s)^{q-1}\left\|f\left(s, u(s), u_{s}\right)\right\|_{\mathscr{D}} \mathrm{d} s+\frac{1}{\Gamma(q)} \int_{0}^{t}(t-s)^{q-1}\left\|B \mu_{u}(s)\right\|_{\mathscr{D}} \mathrm{d} s \\
& \leq \frac{M_{0} T^{* q}}{\Gamma(q+1)}+\frac{N_{1} M_{1} T^{* q}}{\Gamma(q+1)}=\frac{\left(M_{0}+N_{1} M_{1}\right) T^{* q}}{\Gamma(q+1)} .
\end{aligned}
$$

Then, this together with (24) indicates

$\|(\Psi u)(t)\|$

$$
\begin{aligned}
& \leq\|\phi(0)\|_{\mathscr{D}}+\|M(0, u)\|_{\mathscr{D}}+\left\|\Pi\left(t, u, \mu_{u}\right)\right\|_{\mathscr{D}}+\int_{0}^{t} \varphi(t-s)\left(\|\phi(0)\|_{\mathscr{D}}+\|M(0, u)\|_{\mathscr{D}}+\left\|\Pi\left(s, u, \mu_{u}\right)\right\|_{\mathscr{D}}\right) \mathrm{d} s \\
& \leq\|\phi(0)\|_{\mathscr{D}}+G+\frac{\left(M_{0}+N_{1} M_{1}\right) T^{* q}}{\Gamma(q+1)}+\|\varphi\|_{L^{1}(I)}\left(\|\phi(0)\|_{\mathscr{D}}+G+\frac{\left(M_{0}+N_{1} M_{1}\right) T^{* q}}{\Gamma(q+1)}\right) \\
& \leq\left(\|\phi(0)\|_{\mathscr{D}}+G\right)\left(\|\varphi\|_{L^{1}(I)}+1\right)+\frac{\left(M_{0}+N_{1} M_{1}\right)\left(\|\varphi\|_{L^{1}(I)}+1\right)}{\Gamma(q+1)} T^{* q} \leq R_{0} .
\end{aligned}
$$

On the contrary,

$$
\begin{aligned}
\left\|(\Psi u)_{t}\right\|_{L[-r, 0]} & =\int_{-r}^{0}\left\|(\Psi u)_{t}(\theta)\right\| \mathrm{d} \theta \\
& = \begin{cases}\int_{t-r}^{0}\|\phi(s)\| \mathrm{d} s+\int_{0}^{t}\|(\Psi u)(s)\| \mathrm{d} s, & t \leq r, \\
\int_{t-r}^{t}\|(\Psi u)(s)\| \mathrm{d} s, & t \geq r,\end{cases}
\end{aligned}
$$

which infers that

$$
\left\|(\Psi u)_{t}\right\|_{L[-r, 0]} \leq r\|\phi\|_{r}+r\|\Psi u\|_{C([0, t], X)} .
$$

Therefore, we can obtain

$$
\sup _{t \in\left[0, T^{*}\right]}\left\|(\Psi u)_{t}\right\|_{L[-r, 0]} \leq r\left(\|\phi\|_{r}+R_{0}\right) .
$$

It is obvious that $(\Psi u)(t)=\phi(t)-M(t, u)$ for any $u \in \Omega, t \in[-r, 0]$. Then, we conclude that $\Psi(\Omega) \subseteq \Omega$.

Next, we shall demonstrate that $\Psi: \Omega \longrightarrow \Omega$ is equicontinuous on $J$. For arbitrary $u \in \Omega$ and $\tau_{1}, \tau_{2} \in J=$ $\left[-r, T^{*}\right]$ with $\tau_{1}<\tau_{2}$, we present the following discussions.

$$
\begin{aligned}
(\Psi u)\left(\tau_{2}\right)-(\Psi u)\left(\tau_{1}\right)= & \Pi\left(\tau_{2}, u, \mu_{u}\right)-\Pi\left(\tau_{1}, u, \mu_{u}\right) \\
& +\int_{0}^{\tau_{2}} \dot{\mathfrak{J}}\left(\tau_{2}-s\right)(\phi(0)-M(0, u)) \mathrm{d} s-\int_{0}^{\tau_{1}} \dot{\mathfrak{J}}\left(\tau_{1}-s\right)(\phi(0)-M(0, u)) \mathrm{d} s \\
& +\int_{0}^{\tau_{2}} \dot{\mathfrak{J}}\left(\tau_{2}-s\right) \Pi\left(s, u, \mu_{u}\right) \mathrm{d} s-\int_{0}^{\tau_{1}} \dot{\mathfrak{J}}\left(\tau_{1}-s\right) \Pi\left(s, u, \mu_{u}\right) \mathrm{d} s . \\
& \left\|(\Psi u)\left(\tau_{2}\right)-(\Psi u)\left(\tau_{1}\right)\right\| \leq\left\|\mathscr{B}_{1}\right\|+\left\|\mathscr{B}_{2}\right\|+\left\|\mathscr{B}_{3}\right\|,
\end{aligned}
$$

Clearly, 
where

$$
\begin{aligned}
& \mathscr{B}_{1}=\Pi\left(\tau_{2}, u, \mu_{u}\right)-\Pi\left(\tau_{1}, u, \mu_{u}\right), \\
& \mathscr{B}_{2}=\int_{0}^{\tau_{2}} \dot{\mathfrak{J}}\left(\tau_{2}-s\right)(\phi(0)-M(0, u)) \mathrm{d} s-\int_{0}^{\tau_{1}} \dot{\mathfrak{I}}\left(\tau_{1}-s\right)(\phi(0)-M(0, u)) \mathrm{d} s, \\
& \mathscr{B}_{3}=\int_{0}^{\tau_{2}} \dot{\mathfrak{J}}\left(\tau_{2}-s\right) \Pi\left(s, u, \mu_{u}\right) \mathrm{d} s-\int_{0}^{\tau_{1}} \dot{\mathfrak{J}}\left(\tau_{1}-s\right) \Pi\left(s, u, \mu_{u}\right) \mathrm{d} s .
\end{aligned}
$$

Next, we shall show that $\left\|\mathscr{B}_{i}\right\| \longrightarrow 0$ is independent of $u \in \Omega$ as $\left|\tau_{1}-\tau_{2}\right| \longrightarrow 0, i=1,2,3$. In fact, with regard to $\mathscr{B}_{1}$, we derive

$\left\|\mathscr{B}_{1}\right\|$

$$
\begin{aligned}
\leq & \left\|\int_{0}^{\tau_{2}}\left(\tau_{2}-s\right)^{q-1} f\left(s, u(s), u_{s}\right) \mathrm{d} s-\int_{0}^{\tau_{1}}\left(\tau_{1}-s\right)^{q-1} f\left(s, u(s), u_{s}\right) \mathrm{d} s\right\|+\left\|\int_{0}^{\tau_{2}}\left(\tau_{2}-s\right)^{q-1} B \mu_{u}(s) \mathrm{d} s-\int_{0}^{\tau_{1}}\left(\tau_{1}-s\right)^{q-1} B \mu_{u}(s) \mathrm{d} s\right\| \\
\leq & \int_{0}^{\tau_{1}}\left[\left(\tau_{1}-s\right)^{q-1}-\left(\tau_{2}-s\right)^{q-1}\right]\left\|f\left(s, u(s), u_{s}\right)\right\|_{\mathscr{D}} \mathrm{d} s+\int_{\tau_{1}}^{\tau_{2}}\left(\tau_{2}-s\right)^{q-1}\left\|f\left(s, u(s), u_{s}\right)\right\|_{\mathscr{D}} \mathrm{d} s \\
& +\int_{0}^{\tau_{1}}\left[\left(\tau_{1}-s\right)^{q-1}-\left(\tau_{2}-s\right)^{q-1}\right]\left\|B \mu_{u}(s)\right\|_{\mathscr{D}} \mathrm{d} s+\int_{\tau_{1}}^{\tau_{2}}\left(\tau_{2}-s\right)^{q-1}\left\|B \mu_{u}(s)\right\|_{\mathscr{D}} \mathrm{d} s \\
\leq & \frac{M_{0}}{q}\left(\tau_{1}^{q}-\tau_{2}^{q}+\left(\tau_{2}-\tau_{1}\right)^{q}\right)+\frac{M_{0}}{q}\left(\tau_{2}-\tau_{1}\right)^{q}+\frac{N_{1} M_{1}}{q}\left(\tau_{1}^{q}-\tau_{2}^{q}+\left(\tau_{2}-\tau_{1}\right)^{q}\right)+\frac{N_{1} M_{1}}{q}\left(\tau_{2}-\tau_{1}\right)^{q} \longrightarrow 0, \text { as }\left|\tau_{1}-\tau_{2}\right| \longrightarrow 0 .
\end{aligned}
$$

Notice that

$$
\begin{aligned}
\mathscr{B}_{2} & =\int_{0}^{\tau_{2}} \dot{\mathfrak{\Im}}\left(\tau_{2}-s\right)(\phi(0)-M(0, u)) \mathrm{d} s-\int_{0}^{\tau_{1}} \dot{\mathfrak{\Im}}\left(\tau_{1}-s\right)(\phi(0)-M(0, u)) \mathrm{d} s \\
& =\int_{0}^{\tau_{2}-\tau_{1}} \dot{\mathfrak{\Im}}\left(\tau_{2}-s\right)(\phi(0)-M(0, u)) \mathrm{d} s+\int_{\tau_{2}-\tau_{1}}^{\tau_{2}} \dot{\mathfrak{\Im}}\left(\tau_{2}-s\right)(\phi(0)-M(0, u)) \mathrm{d} s-\int_{0}^{\tau_{1}} \dot{\mathfrak{\Im}}\left(\tau_{1}-s\right)(\phi(0)-M(0, u)) \mathrm{d} s \\
& =\int_{0}^{\tau_{2}-\tau_{1}} \dot{\mathfrak{J}}\left(\tau_{2}-s\right)(\phi(0)-M(0, u)) \mathrm{d} s .
\end{aligned}
$$

According to Definition 4, we deduce

$$
\begin{aligned}
\left\|\mathscr{B}_{2}\right\| & \leq\left(\|\phi(0)\|_{\mathscr{D}}+\|M(0, u)\|_{\mathscr{D}}\right) \int_{0}^{\tau_{2}-\tau_{1}} \varphi\left(\tau_{2}-s\right) \mathrm{d} s \\
& \leq\left(\|\phi(0)\|_{\mathscr{D}}+G\right) \int_{\tau_{1}}^{\tau_{2}} \varphi(s) \mathrm{d} s \longrightarrow 0 \text {, as }\left|\tau_{1}-\tau_{2}\right| \longrightarrow 0 .
\end{aligned}
$$


From the proof process of Lemma 6 and (32), it follows that

$$
\begin{aligned}
\left\|\mathscr{B}_{3}\right\| & =\left\|\int_{0}^{\tau_{2}} \dot{\mathfrak{J}}\left(\tau_{2}-s\right) \Pi\left(s, u, \mu_{u}\right) \mathrm{d} s-\int_{0}^{\tau_{1}} \dot{\mathfrak{J}}\left(\tau_{1}-s\right) \Pi\left(s, u, \mu_{u}\right) \mathrm{d} s\right\| \\
& \leq\left\|\int_{0}^{\tau_{2}-\tau_{1}} \dot{\mathfrak{J}}\left(\tau_{2}-s\right) \Pi\left(s, u, \mu_{u}\right) \mathrm{d} s+\int_{\tau_{2}-\tau_{1}}^{\tau_{2}} \dot{\mathfrak{J}}\left(\tau_{2}-s\right) \Pi\left(s, u, \mu_{u}\right) \mathrm{d} s-\int_{0}^{\tau_{1}} \dot{\mathfrak{J}}\left(\tau_{1}-s\right) \Pi\left(s, u, \mu_{u}\right) \mathrm{d} s\right\| \\
& \leq\left\|\int_{0}^{\tau_{2}-\tau_{1}} \dot{\mathfrak{J}}\left(\tau_{2}-s\right) \Pi\left(s, u, \mu_{u}\right) \mathrm{d} s+\int_{0}^{\tau_{1}} \dot{\mathfrak{J}}(s) \Pi\left(\tau_{2}-s, u, \mu_{u}\right) \mathrm{d} s-\int_{0}^{\tau_{1}} \dot{\mathfrak{J}}(s) \Pi\left(\tau_{1}-s, u, \mu_{u}\right) \mathrm{d} s\right\| \\
& \leq \int_{0}^{\tau_{2}-\tau_{1}} \varphi\left(\tau_{2}-s\right)\left\|\Pi\left(s, u, \mu_{u}\right)\right\|_{\mathscr{D}} \mathrm{d} s+\int_{0}^{\tau_{1}} \varphi(s)\left\|\Pi\left(\tau_{2}-s, u, \mu_{u}\right)-\Pi\left(\tau_{1}-s, u, \mu_{u}\right)\right\|_{\mathscr{D}} \mathrm{d} s \\
& \leq \int_{\tau_{1}}^{\tau_{2}} \varphi(s) \mathrm{d} s \cdot \frac{\left(M_{0}+N_{1} M_{1}\right) T^{* q}}{\Gamma(q+1)}+\int_{0}^{\tau_{1}} \varphi(s) \mathrm{d} s \cdot \frac{2\left(M_{0}+N_{1} M_{1}\right)}{q}\left(\tau_{2}-\tau_{1}\right)^{q} \longrightarrow 0, \text { as }\left|\tau_{1}-\tau_{2}\right| \longrightarrow 0 .
\end{aligned}
$$

(ii) If $-r \leq \tau_{1}<\tau_{2} \leq 0$, then from the continuity of $\phi(\cdot)$

and (H3) (i), one has

$$
\begin{aligned}
\left\|(\Psi u)\left(\tau_{2}\right)-(\Psi u)\left(\tau_{1}\right)\right\| & =\left\|\phi\left(\tau_{2}\right)-\phi\left(\tau_{1}\right)-\left(M\left(\tau_{2}, u\right)-M\left(\tau_{1}, u\right)\right)\right\| \\
& \leq\left\|\phi\left(\tau_{2}\right)-\phi\left(\tau_{1}\right)\right\|+\left\|M\left(\tau_{2}, u\right)-M\left(\tau_{1}, u\right)\right\| \longrightarrow 0, \text { as }\left|\tau_{1}-\tau_{2}\right| \longrightarrow 0
\end{aligned}
$$

(iii) If $-r \leq \tau_{1}<0<\tau_{2} \leq T^{*}$, then

$$
\begin{aligned}
& \left\|(\Psi u)\left(\tau_{2}\right)-(\Psi u)\left(\tau_{1}\right)\right\| \\
& \quad \leq\left\|(\Psi u)\left(\tau_{2}\right)-(\Psi u)(0)\right\|+\left\|(\Psi u)(0)-(\Psi u)\left(\tau_{1}\right)\right\| \\
& \quad \leq\left\|\Pi\left(\tau_{2}, u, \mu_{u}\right)+\int_{0}^{\tau_{2}} \dot{\Im}\left(\tau_{2}-s\right)\left(\phi(0)-M(0, u)+\Pi\left(s, u, \mu_{u}\right)\right) \mathrm{d} s\right\|+\left\|(\phi(0)-M(0, u))-\phi\left(\tau_{1}\right)-M\left(\tau_{1}, u\right)\right\| \\
& \quad \leq \frac{M_{0}+N_{1} M_{1}}{\Gamma(q+1)} \tau_{2}^{q}+\left(\|\phi(0)\|_{D}+\|M(0, u)\|_{\mathscr{D}}+\left\|\Pi\left(s, u, \mu_{u}\right)\right\|_{\mathscr{D}}\right) \int_{0}^{\tau_{2}} \varphi\left(\tau_{2}-s\right) \mathrm{d} s+\left\|\phi(0)-\phi\left(\tau_{1}\right)\right\|+\left\|M(0, u)-M\left(\tau_{1}, u\right)\right\| \\
& \quad \leq \frac{M_{0}+N_{1} M_{1}}{\Gamma(q+1)} \tau_{2}^{q}+\left(\|\phi(0)\|_{\mathscr{D}}+G+\frac{M_{0}+N_{1} M_{1}}{\Gamma(q+1)} T^{q}\right) \int_{0}^{\tau_{2}} \varphi(s) \mathrm{d} s+\left\|\phi(0)-\phi\left(\tau_{1}\right)\right\|+\left\|M(0, u)-M\left(\tau_{1}, u\right)\right\| \\
& \quad \longrightarrow 0, \text { as }\left|\tau_{1}-\tau_{2}\right| \longrightarrow 0 .
\end{aligned}
$$

Thus, it implies that $\left\|(\Psi u)\left(\tau_{2}\right)-(\Psi u)\left(\tau_{1}\right)\right\| \longrightarrow 0$, as $\left|\tau_{1}-\tau_{2}\right| \longrightarrow 0$, for all $u \in \Omega$. Consequently, $\Psi: \Omega \longrightarrow \Omega$ is equicontinuous on $J$.

Lemma 8. Let conditions (H1) (i), (H2), (H3) (i), and (H4) be satisfied. Then, the operator $\Psi: \Omega \longrightarrow \Omega$ is continuous.

Proof. It follows from Lemma 7 that $\Psi(\Omega) \subseteq \Omega$. Next, we show that $\Psi$ is continuous. Let $\left\{v_{n}\right\}$ be a sequence such that $v_{n} \longrightarrow v$ in $\Omega$ as $n \longrightarrow \infty$.
In view of condition (H1) (i) and Lebesgue dominated convergence theorem, we have

$$
\begin{array}{r}
\int_{0}^{t}(t-s)^{q-1}\left\|f\left(s, v_{n}(s),\left(v_{n}\right)_{s}\right)-f\left(s, v(s), v_{s}\right)\right\|_{\mathscr{D}} \mathrm{d} s \\
\forall t \in\left[0, T^{*}\right], \text { as } n \longrightarrow+\infty .
\end{array}
$$

Therefore, this together with the continuity of $M(0, \cdot)$ implies 


$$
\begin{aligned}
& \left\|B \mu_{v_{n}}(s)-B \mu_{v}(s)\right\|_{\mathscr{D}} \\
& \leq N_{1} N_{2}\left[\left\|M\left(0, v_{n}\right)-M(0, v)\right\|_{\mathscr{D}}+\left\|\Pi_{f}\left(T^{*}, v_{n}\right)-\Pi_{f}\left(T^{*}, v\right)\right\|_{\mathscr{D}}\right. \\
& \left.\quad+\int_{0}^{T^{*}} \varphi\left(T^{*}-s\right)\left\|M\left(0, v_{n}\right)-M(0, v)\right\|_{\mathscr{D}}+\left\|\Pi_{f}\left(s, v_{n}\right)-\Pi_{f}(s, v)\right\|_{\mathscr{D}} \mathrm{d} s\right] \\
& \leq N_{1} N_{2}\left[\left\|M\left(0, v_{n}\right)-M(0, v)\right\|_{\mathscr{D}}+\int_{0}^{T^{*}}\left(T^{*}-s\right)^{q-1}\left\|f\left(s, v_{n}(s),\left(v_{n}\right)_{s}\right)-f\left(s, v(s), v_{s}\right)\right\|_{\mathscr{D}} \mathrm{d} s\right. \\
& \left.\quad+\int_{0}^{T^{*}} \varphi\left(T^{*}-s\right)\left(\left\|M\left(0, v_{n}\right)-M(0, v)\right\|_{\mathscr{D}}+\int_{0}^{s}(s-\eta)^{q-1}\left\|f\left(\eta, v_{n}(\eta),\left(v_{n}\right)_{\eta}\right)-f\left(\eta, v(\eta), v_{\eta}\right)\right\|_{\mathscr{D}} \mathrm{d} \eta\right) \mathrm{d} s\right] \\
& \quad \longrightarrow 0, \text { as } n \longrightarrow+\infty .
\end{aligned}
$$

Consequently, for each $t \in\left[0, T^{*}\right]$, we have

$$
\begin{aligned}
& \left\|\left(\Psi v_{n}\right)(t)-(\Psi v)(t)\right\| \\
& \leq\left\|\Pi\left(t, v_{n}, \mu_{v_{n}}\right)-\Pi\left(t, v, \mu_{v}\right)\right\|_{\mathscr{D}}+\int_{0}^{t}\left\|\dot{\mathfrak{J}}(t-s)\left(\Pi\left(s, v_{n}, \mu_{v_{n}}\right)-\Pi\left(s, v, \mu_{v}\right)\right)\right\| \mathrm{d} s \\
& \leq \int_{0}^{t} \int_{0}^{t}(t-s)^{q-1}\left\|f\left(s, v_{n}(s),\left(v_{n}\right)_{s}\right)-f\left(s, v(s), v_{s}\right)_{\mathscr{D}}\right\| \mathrm{d} s+\int_{0}^{t}(t-s)^{q-1}\left\|B \mu_{v_{n}}(s)-B \mu_{v}(s)\right\|_{\mathscr{D}} \mathrm{d} s \\
& \quad+\int_{0}^{t} \varphi(t-s)\left(\int_{0}^{s}(s-\eta)^{q-1}\left\|f\left(\eta, v_{n}(\eta),\left(v_{n}\right)_{\eta}\right)-f\left(\eta, v(\eta), v_{\eta}\right)\right\|_{\mathscr{D}} \mathrm{d} \eta\right) \mathrm{d} s \\
& \quad+\int_{0}^{t} \varphi(t-s)\left(\int_{0}^{s}(s-\eta)^{q-1}\left\|B \mu_{v_{n}}(\eta)-B \mu_{v}(\eta)\right\|_{\mathscr{D}} \mathrm{d} \eta\right) \longrightarrow 0, \text { as } n \longrightarrow+\infty .
\end{aligned}
$$

From the analogous proof of equicontinuous for operator $\Psi$ in Lemma 7 and the well-known Ascoli-Arzelà theorem, it is not difficult to obtain $\left\|\Psi v_{n}-\Psi v\right\|_{C(J, X)} \longrightarrow 0$, as $n \longrightarrow+\infty$, i.e., $\Psi$ is continuous on $\Omega$. This completes the proof.

We present now our main results of this paper.

Theorem 1. If assumptions (H1)-(H4) hold, then the fractional evolution (1) is completely controllable on I.

Proof. Consider the operator $\Psi$ defined as (28). In view of Lemma 1, it is enough to prove that when using the control $\mu_{u}$, the operator $\Psi$ has a fixed point $u(\cdot)$ which is exactly a mild solution of (1) on $J$. From the verified fact $u\left(T^{*}\right)=(\Psi u)\left(T^{*}\right)=u_{1}$, it follows that the control $\mu_{u}$ steers system (1) from the initial function $\phi$ to $u_{1}$ in finite time $T^{*}$. The complete controllability on $I$ of system (1) is thus proved. To this end, we shall take advantage of Mönch fixed point theorem. The continuity of operator $\Psi: \Omega \longrightarrow \Omega$ is given by Lemma 8 . In the next step, we demonstrate that Mönch's condition holds for operator $\Psi: \Omega \longrightarrow \Omega$.

Let $B=\overline{c o} \Psi(\Omega)$. It is not difficult to check that $\Psi(B) \subseteq B$. Assume that bounded set $D_{0} \subset B$ is countable and $D_{0} \subset \overline{c o}\left(\left\{u_{0}\right\} \cup \Psi\left(D_{0}\right)\right)$, and we shall prove that $\gamma\left(D_{0}\right)=0$.
From Lemma 7, it is easy to derive that $\Psi\left(D_{0}\right)$ is equicontinuous on $J$. Notice that $D_{0} \subset \overline{c o}\left(\left\{u_{0}\right\} \cup \Psi\left(D_{0}\right)\right)$, so $D_{0}$ is also equicontinuous on $J$.

For any $u \in D_{0}$, let

$$
(\Psi u)(t)= \begin{cases}\left(\Psi_{1} u\right)(t)+\left(\Psi_{2} u\right)(t), & t \in\left[0, T^{*}\right], \\ \phi(t)-M(t, u), & t \in[-r, 0],\end{cases}
$$

where

$$
\begin{aligned}
& \left(\Psi_{1} u\right)(t)=\phi(0)-M(0, u)+\Pi\left(t, u, \mu_{u}\right), \\
& \left(\Psi_{2} u\right)(t)=\int_{0}^{t} \dot{\mathfrak{\Im}}(t-s)\left(\phi(0)-M(0, u)+\Pi\left(s, u, \mu_{u}\right)\right) \mathrm{d} s
\end{aligned}
$$

No loss of generality, suppose $D_{0}=\left\{w_{n}\right\}_{n=1}^{\infty}$. From hypothesis (H1) (ii) and Lemma 5, for any $s \in I$, it follows that

$$
\begin{aligned}
\gamma\left(\left\{f\left(s, w_{n}(s),\left(w_{n}\right)_{s}\right)\right\}\right) & \leq m(s)\left(\left(\gamma\left(\left\{w_{n}(s)\right\}\right)\right)+\gamma\left(\left\{\left(w_{n}\right)_{s}\right\}\right)\right) \\
& \leq m(s)\left(\left(\gamma\left(\left\{w_{n}(s)\right\}\right)\right)+r \gamma\left(\left\{w_{n}\right\}\right)\right) \\
& \leq m(s)\left(\gamma\left(D_{0}(s)\right)+r \gamma\left(D_{0}\right)\right) \\
& \leq m(s)(r+1) \gamma\left(D_{0}\right) .
\end{aligned}
$$

Then, this indicates from Lemma 2 and Hölder inequality that 


$$
\begin{aligned}
\gamma\left(\left\{\Pi_{f}\left(t, w_{n}\right)\right\}\right) & =\gamma\left(\left\{\frac{1}{\Gamma(q)} \int_{0}^{t}(t-s)^{q-1} f\left(s, w_{n}(s),\left(w_{n}\right)_{s}\right) \mathrm{d} s\right\}\right) \\
& \leq \frac{2}{\Gamma(q)}\left(\int_{0}^{t}(t-s)^{q-1} \gamma\left(\left\{f\left(s, w_{n}(s),\left(w_{n}\right) s\right)\right\}\right) \mathrm{d} s\right) \\
& \leq \frac{2(r+1)}{\Gamma(q)} \int_{0}^{t}(t-s)^{q-1} m(s) \mathrm{d} s \cdot \gamma\left(\left\{w_{n}\right\}\right) \\
& \leq \frac{2(r+1)}{\Gamma(q)}\left(\int_{0}^{t}\left[(t-s)^{q-1}\right]\left(1 / 1-q_{1}\right) \mathrm{d} s\right)^{1-q_{1}}\left(\int_{0}^{t} m(s)\left(1 / q_{1}\right) \mathrm{d} s\right)^{q_{1}} \cdot \gamma\left(\left\{w_{n}\right\}\right) \\
& \leq \frac{2(r+1)}{\Gamma(q)}\left(\frac{1-q_{1}}{q-q_{1}}\right)^{1-q_{1}} t^{q-q_{1}}\|m\|_{\left.L^{\left(1 / q_{1}\right.}\right)} \cdot \gamma\left(\left\{w_{n}\right\}\right) \\
& \leq \lambda T^{* q} \gamma\left(D_{0}\right), \quad t \in\left[0, T^{*}\right],
\end{aligned}
$$

which together with Lemma 2, (H2) (ii), and (H3) (i) implies

$$
\begin{aligned}
& \gamma\left(\left\{\mu_{w_{n}}(s)\right\}\right) \\
& \quad \leq p(s)\left(\gamma\left(\left\{M\left(0, w_{n}\right)\right\}\right)+\gamma\left(\left\{\Pi_{f}\left(T^{*}, w_{n}\right)\right\}\right)+2 \int_{0}^{T^{*}} \varphi\left(T^{*}-s\right)\left(\gamma\left(\left\{M\left(0, w_{n}\right)\right\}\right)+\gamma\left(\left\{\Pi_{f}\left(s, w_{n}\right)\right\}\right)\right) \mathrm{d} s\right) \\
& \quad \leq p(s)\left(k \gamma\left\{w_{n}\right\}+\lambda T^{* q} \gamma\left(\left\{w_{n}\right\}\right)+2\|\varphi\|_{L^{1}(I)}\left(k \gamma\left\{w_{n}\right\}+\lambda T^{* q} \gamma\left(\left\{w_{n}\right\}\right)\right)\right) \\
& \quad \leq p(s)\left(k+\lambda T^{* q}\right)\left(1+2\|\varphi\|_{L^{1}(I)}\right) \gamma\left(D_{0}\right), \quad s \in\left[0, T^{*}\right] .
\end{aligned}
$$

Thus, by using Hölder inequality again, one has

$$
\begin{aligned}
\gamma\left(\left\{\Pi_{B}\left(t, \mu_{w_{n}}\right)\right\}\right) & \leq \frac{2 N_{1}}{\Gamma(q)} \int_{0}^{t}(t-s)^{q-1} \gamma\left(\left\{\mu_{w_{n}}(s)\right\}\right) \mathrm{d} s \\
& \leq \frac{2 N_{1}\left(k+\lambda T^{* q}\right)\left(1+2\|\varphi\|_{L^{1}(I)}\right)}{\Gamma(q)} \int_{0}^{t}(t-s)^{q-1} p(s) \mathrm{d} s \gamma\left(D_{0}\right) \\
& \leq \frac{2 N_{1} L\left(k+\lambda T^{* q}\right)\left(1+2\|\varphi\|_{L^{1}(I)}\right)}{\Gamma(q)} \gamma\left(D_{0}\right), \quad t \in\left[0, T^{*}\right] .
\end{aligned}
$$

Consequently, from the abovementioned, we can derive

$$
\begin{aligned}
\gamma\left(\Psi_{1}\left(D_{0}\right)(t)\right) & \leq \gamma\left(\left\{M\left(0, w_{n}\right)\right\}\right)+\gamma\left(\left\{\Pi_{f}\left(t, w_{n}\right)\right\}\right)+\gamma\left(\left\{\Pi_{B}\left(t, \mu_{w_{n}}\right)\right\}\right) \\
& \leq k \gamma\left(D_{0}\right)+\lambda T^{* q} \gamma\left(D_{0}\right)+\frac{2 N_{1} L\left(k+\lambda T^{* q}\right)\left(1+2\|\varphi\|_{L^{1}(I)}\right)}{\Gamma(q)} \gamma\left(D_{0}\right) \\
& \leq\left(k+\lambda T^{* q}\right)\left(1+\frac{2 N_{1} L\left(1+2\|\varphi\|_{L^{1}(I)}\right)}{\Gamma(q)}\right) \gamma\left(D_{0}\right) \\
& \leq\left(\lambda M T^{* q}+k M\right) \gamma\left(D_{0}\right) .
\end{aligned}
$$


For $t \in\left[0, T^{*}\right]$, also in view of Lemma 2 , one obtains

$$
\begin{aligned}
\gamma\left(\Psi_{2}\left(D_{0}\right)(t)\right) & =\gamma\left(\left\{\int_{0}^{t} \dot{\mathfrak{\Im}}(t-s)\left(\phi(0)-M\left(0, w_{n}\right)+\Pi\left(s, w_{n}, \mu_{w_{n}}\right)\right) \mathrm{d} s\right\}\right) \\
& \leq 2 \int_{0}^{t} \gamma\left(\left\{\dot{\mathfrak{J}}(t-s)\left(\phi(0)-M\left(0, w_{n}\right)+\Pi\left(s, w_{n}, \mu_{w_{n}}\right)\right)\right\}\right) \mathrm{d} s \\
& \leq 2 \int_{0}^{t} \varphi(t-s) \gamma\left(\Psi_{1}\left(D_{0}\right)(s)\right) \mathrm{d} s \\
& \leq 2\|\varphi\|_{L^{1}(I)}\left(\lambda M T^{* q}+k M\right) \gamma\left(D_{0}\right) .
\end{aligned}
$$

Then, by (55) and (56), we can infer

$$
\begin{aligned}
\gamma\left(\Psi\left(D_{0}\right)(t)\right) \leq & \gamma\left(\Psi_{1}\left(D_{0}\right)(t)\right)+\gamma\left(\Psi_{2}\left(D_{0}\right)(t)\right) \\
\leq & \left(\lambda M T^{* q}+k M\right) \gamma\left(D_{0}\right) \\
& +2\|\varphi\|_{L^{1}(I)}\left(\lambda M T^{* q}+k M\right) \gamma\left(D_{0}\right) \\
\leq & \left(\lambda M\left(1+2\|\varphi\|_{L^{1}(I)}\right) T^{* q}\right. \\
& \left.+k M\left(1+2\|\varphi\|_{L^{1}(I)}\right)\right) \gamma\left(D_{0}\right), \quad t \in\left[0, T^{*}\right] .
\end{aligned}
$$

Besides, for $t \in[-r, 0]$, we have from (H3) (i) that

$$
\begin{aligned}
\gamma\left(\Psi\left(D_{0}\right)(t)\right) & \leq \gamma\left(\left\{\phi(t)-M\left(t, w_{n}\right)\right\}\right) \\
& \leq \gamma\left(\left\{M\left(t, w_{n}\right)\right\}\right) \leq k \gamma\left(\left\{w_{n}\right\}\right)<\gamma\left(D_{0}\right) .
\end{aligned}
$$

On the contrary, from the equicontinuity of $\Psi\left(D_{0}\right)$ on $J$, it follows that

$$
\gamma\left(\Psi\left(D_{0}\right)\right)=\max _{t \in J} \gamma\left(\left(\Psi D_{0}\right)(t)\right) .
$$

Consequently, by (24) and (57)-(59), one can derive

$$
\gamma\left(D_{0}\right) \leq \gamma\left(\overline{c o}\left(\left\{u_{0}\right\} \cup \Psi\left(D_{0}\right)\right)\right) \leq \gamma\left(\Psi\left(D_{0}\right)\right)<\gamma\left(D_{0}\right),
$$

which deduces $\gamma\left(D_{0}\right)=0$. Due to Lemma 2 (I) (i), we know that $D_{0}$ is relatively compact. Then, from Lemma $3, \Psi$ has at least one fixed point $u \in B$. This shows that the complete controllability on $I$ for system (1) is valid. The proof is now completed.

Remark 3. (i) Different from some papers [21, 25, 44] utilizing the definition of mild solution by probability density functions which are initially presented by El-Borai [3], the way of making use of differentiable resolvent operators to define mild solution in this paper can avoid the complexity of definitions and properties related to the probability density functions and its associated characteristic solution operators; sometimes, the limitation of fractional order $q$ lying in $(0,1)$ due to the probability density functions can be extricated. (ii) In a comparative way, nonlocal item in this paper has better application effect in physics. In practical applications, it may be given by $M(t, u)=$ $\sum_{i=1}^{k} c_{i} u\left(\tau_{i}+t\right), \quad t \in[-r, 0]$, where $c_{i}(i=1,2, \ldots, k)$ is a given constant and $0<\tau_{1}<\tau_{2}<\cdots<\tau_{n} \leq T$. At time $t=0$, we have $M(0, u)=\sum_{i=1}^{k} c_{i} u\left(\tau_{i}\right)$, which is exactly the case in [44].

\section{An Example}

As an application of our abstract results, we consider the following fractional partial differential nonlocal evolution equations with delay of the form:

$$
\begin{cases}\frac{\partial^{(1 / 3)}}{\partial t^{(1 / 3)}} u(t, \tau)=\frac{\partial}{\partial \tau} u(t, \tau)+\frac{\vartheta(t) u_{t}(\tau)}{2+\left|u_{t}(\tau)\right|}+\int_{-r}^{t} \eta(t-s) u(s, \tau) d s+\delta(\tau) \mu(t, \tau), & (t, \tau) \in[0, T] \times(0, \pi), \\ u(t, 0)=u(t, \pi)=0, & t \in[0, T], \\ u(t, \tau)+\sum_{i=1}^{l} m_{i} \sin \left(u\left(c_{i}+t, \tau\right)\right)=\phi(t, \tau), & (t, \tau) \in[-r, 0] \times[0, \pi], 0<c_{1}<c_{2}<\cdots<c_{l}<T,\end{cases}
$$

where $\vartheta \in C([0, T], R), \eta \in L((-r, T+r), R), \delta$ denotes the characteristic function of some subinterval $D \subset[0, \pi]$, $\mu \in C([0, T] \times[0, \pi], R)$ and satisfies $\mu(t, 0)=\mu(t, \pi)=0$ for $t \in[0, T] . m_{i}$ is a fixed constant for $i=1,2, \ldots, l$ satisfying $\sum_{i=1}^{l}\left|m_{i}\right|<1 . \phi \in C([-r, 0] \times[0, \pi], X)$, which satisfies $\phi(t, 0)=\phi(t, \pi)=0$ for $t \in[-r, 0], X=C([0, \pi], R)$, and $\mathscr{D}=\left\{u \in X: u^{\prime} \in X, u(0)=u(\pi)=0\right\}, A u=u^{\prime}$ for $u \in \mathscr{D}$. Thus, $A$ is an infinitesimal generator of a noncompact semigroup $\{T(t): t \geq 0\}$ which is given by $T(t) u(s)=u(t+$ $s)$ for $u \in X$. Let 


$$
\begin{aligned}
D^{(1 / 3)} u(t)(\tau) & =\frac{\partial^{(1 / 3)}}{\partial t^{(1 / 3)}} u(t, \tau) \\
f\left(t, u(t), u_{t}\right)(\tau) & =\frac{\vartheta(t) u_{t}(\tau)}{2+\left|u_{t}(\tau)\right|}+\int_{-r}^{t} \eta(t-s) u(s, \tau) d s \\
u(t)(\tau) & =u(t, \tau), \\
B \mu(t)(\tau) & =\delta(\tau) \mu(t, \tau), \\
\phi(t)(\tau) & =\phi(t, \tau), \\
M(t, u)(\tau) & =\sum_{i=1}^{l} m_{i} \sin \left(u\left(c_{i}+t, \tau\right)\right), \\
\sum_{i=1}^{l}\left|m_{i}\right| & <1, \quad 0<c_{1}<c_{2}<\cdots<c_{l}<T .
\end{aligned}
$$

Under these assumptions, partial system (61) can be regarded as a control problem of the abstract form:

$$
\begin{cases}D^{q} u(t)=A u(t)+f\left(t, u(t), u_{t}\right)+B \mu(t), & \text { a.e.t } \in I:=[0, T], \\ u(t)+M(t, u)=\phi(t), & t \in[-r, 0] .\end{cases}
$$

For any $t \in[-r, 0]$ and for any bounded set $D \subset C([-r, T], X)$, we have that $\gamma(M(t, D)) \leq$ $\sum_{i=1}^{l}\left|m_{i}\right| \gamma(D)<\gamma(D)$, which indicates that $M(t, \cdot)$ : $C([-r, T], X) \longrightarrow X$ is a strict $k$-set contraction with $k=\sum_{i=1}^{l}\left|m_{i}\right|<1$. In addition, it is not difficult to verify that $f, B$, and $M$ satisfy other assumptions of Theorem 1 . Thus, system (61) is completely controllable on $[0, T]$.

\section{Conclusions}

In this work, complete controllability of a class of semilinear fractional nonlocal evolution systems with finite delay in Banach spaces is investigated by using properties of resolvent operators, theory of measure of noncompactness, and Mönch fixed point theorem. Under a weaker concept of complete controllability and a proper complete space we introduced, the controllability results of the addressed system are obtained without Lipschitz continuity and other growth conditions imposed on the nonlinearity and nonlocal item. In fact, the nonlinear function is only supposed to be continuous. Then, we improve and generalize some analogous results of fractional evolution systems such as $[11,21-25]$.

\section{Data Availability}

No data were used to support this study.

\section{Conflicts of Interest}

The author declares that there are no conflicts of interest.

\section{Acknowledgments}

This research was supported by the National Natural Science Foundation of China under Grant 61873150 and a project of Shandong Province Higher Educational Science and Technology Program of China under Grant J18KA233 hosted by the author Daliang Zhao.

\section{References}

[1] P. Chen, X. Zhang, and Y. Li, "Existence and approximate controllability of fractional evolution equations with nonlocal conditions via resolvent operators," Fractional Calculus and Applied Analysis, vol. 23, no. 1, pp. 268-291, 2020.

[2] P. Chen, X. Zhang, and Y. Li, "Cauchy problem for fractional non-autonomous evolution equations," Banach Journal of Mathematical Analysis, vol. 14, no. 2, pp. 559-584, 2020.

[3] M. M. El-Borai, "Some probability densities and fundamental solutions of fractional evolution equations," Chaos, Solitons \& Fractals, vol. 14, no. 3, pp. 433-440, 2002.

[4] K. S. Miller and B. Ross, An Introduction to the Fractional Calculus and Fractional Differential Equations, A WileyInterscience Publication, John Wiley, Sons, Inc., New York, NY, USA, 1993.

[5] I. Podlubny, Fractional Differential Equations, Mathematics in Science and Engineering, Academic Press, New York, NY, USA, 1999.

[6] T. Qi, Y. Liu, and Y. Cui, "Existence of solutions for a class of coupled fractional differential systems with nonlocal boundary conditions," Journal of Function Spaces, vol. 2017, Article ID 6703860, 9 pages, 2017.

[7] Y. Tian and S. Zhao, "Existence of solutions for perturbed fractional equations with two competing weighted nonlinear terms," Boundary Value Problems, vol. 2018, no. 1, p. 154, 2018.

[8] Y. Tian, "Some results on the eigenvalue problem for a fractional elliptic equation," Boundary Value Problems, vol. 2019, no. 1, p. 13, 2019.

[9] Y. Wang, Y. Liu, and Y. Cui, "Infinitely many solutions for impulsive fractional boundary value problem with $\mathrm{p}$-Laplacian," Boundary Value Problems, vol. 2018, no. 1, p. 94, 2018.

[10] H. Cheng and R. Yuan, "Stability of traveling wave fronts for nonlocal diffusion equation with delayed nonlocal response," Taiwanese Journal of Mathematics, vol. 20, no. 4, pp. 801-822, 2016.

[11] A. Debbouche and D. Baleanu, "Controllability of fractional evolution nonlocal impulsive quasilinear delay integro-differential systems," Computers \& Mathematics with Applications, vol. 62, no. 3, pp. 1442-1450, 2011.

[12] W. Hu, Q. Zhu, and H. R. Karimi, "Some improved razumikhin stability criteria for impulsive stochastic delay differential systems," IEEE Transactions on Automatic Control, vol. 64, no. 12, pp. 5207-5213, 2019.

[13] X. Li, X. Fu, and R. Rakkiyappan, "Delay-dependent stability analysis for a class of dynamical systems with leakage delay and nonlinear perturbations," Applied Mathematics and Computation, vol. 226, pp. 10-19, 2014.

[14] X. Li, J. Shen, and R. Rakkiyappan, "Persistent impulsive effects on stability of functional differential equations with finite or infinite delay," Applied Mathematics and Computation, vol. 329, pp. 14-22, 2018.

[15] Y. Liu, Y. Zheng, H. Li, F. E. Alsaadi, and B. Ahmad, "Control design for output tracking of delayed Boolean control 
networks," Journal of Computational and Applied Mathematics, vol. 327, pp. 188-195, 2018.

[16] I. Stamova, T. Stamov, and X. Li, "Global exponential stability of a class of impulsive cellular neural networks with supremums," International Journal of Adaptive Control and Signal Processing, vol. 28, no. 11, pp. 1227-1239, 2014.

[17] D. Yang, X. Li, J. Shen, and Z. Zhou, "State-dependent switching control of delayed switched systems with stable and unstable modes," Mathematical Methods in the Applied Sciences, vol. 41, no. 16, pp. 6968-6983, 2018.

[18] Q. Zhu, "Stability analysis of stochastic delay differential equations with Lévy noise," Systems \& Control Letters, vol. 118, pp. 62-68, 2018.

[19] Q. Zhu, "Stabilization of stochastic nonlinear delay systems with exogenous disturbances and the event-triggered feedback control," IEEE Transactions on Automatic Control, vol. 64, no. 9, pp. 3764-3771, 2019.

[20] Q. Zhu and T. Huang, "Stability analysis for a class of stochastic delay nonlinear systems driven by G-Brownian motion," Systems \& Control Letters, vol. 140, Article ID 104699, 2020.

[21] J. Du, W. Jiang, D. Pang, and A. U. K. Niazi, "Controllability for a new class of fractional neutral integro-differential evolution equations with infinite delay and nonlocal conditions," Advances in Difference Equations, vol. 139, pp. 1-22, 2017.

[22] R. J. Nirmala, K. Balachandran, L. Rodríguez-Germa, and J. J. Trujillo, "Controllability of nonlinear fractional delay dynamical systems," Reports on Mathematical Physics, vol. 77, no. 1, pp. 87-104, 2016.

[23] Z. Tai, "Controllability of fractional impulsive neutral integrodifferential systems with a nonlocal Cauchy condition in Banach spaces," Applied Mathematics Letters, vol. 24, no. 12, pp. 2158-2161, 2011.

[24] Z. Tai and S. Lun, "On controllability of fractional impulsive neutral infinite delay evolution integrodifferential systems in Banach spaces," Applied Mathematics Letters, vol. 25, no. 2, pp. 104-110, 2012.

[25] J. Wang and Y. Zhou, "Complete controllability of fractional evolution systems," Communications in Nonlinear Science and Numerical Simulation, vol. 17, no. 11, pp. 4346-4355, 2012.

[26] D. Zhao, Y. Liu, and X. Li, "Controllability for a class of semilinear fractional evolution systems via resolvent operators," Communications on Pure \& Applied Analysis, vol. 18, no. 1, pp. 455-478, 2019.

[27] X. Ding, H. Li, Q. Yang, Y. Zhou, A. Alsaedi, and F. E. Alsaadi, "Stochastic stability and stabilization of $\mathrm{n}$-person random evolutionary Boolean games," Applied Mathematics and Computation, vol. 306, pp. 1-12, 2017.

[28] F.-F. Jin and B.-Z. Guo, "Performance boundary output tracking for one-dimensional heat equation with boundary unmatched disturbance," Automatica, vol. 96, pp. 1-10, 2018.

[29] P. Li, X. Li, and J. Cao, "Input-to-state stability of nonlinear switched systems via lyapunov method involving indefinite derivative," Complexity, vol. 2018, Article ID 8701219, 8 pages, 2018.

[30] H. Li, X. Xu, and X. Ding, "Finite-time stability analysis of stochastic switched boolean networks with impulsive effect," Applied Mathematics and Computation, vol. 347, pp. 557-565, 2019.

[31] X. Li, X. Yang, and T. Huang, "Persistence of delayed cooperative models: impulsive control method," Applied Mathematics and Computation, vol. 342, pp. 130-146, 2019.
[32] S. Liang, G. Zhao, H. Li, and X. Ding, "Structural stability analysis of gene regulatory networks modeled by Boolean networks," Mathematical Methods in the Applied Sciences, vol. 42, no. 7, pp. 2221-2230, 2019.

[33] J. Liu and X. Li, "Impulsive stabilization of high-order nonlinear retarded differential equations," Applications of Mathematics, vol. 58, no. 3, pp. 347-367, 2013.

[34] Y. Liu and D. O'Regan, "Controllability of impulsive functional differential systems with nonlocal conditions," Electronic Journal of Differential Equations, vol. 2013, p. 194, 2013.

[35] X. Xu, H. Li, Y. Li, and F. E. Alsaadi, "Output tracking control of Boolean control networks with impulsive effects," Mathematical Methods in the Applied Sciences, vol. 41, no. 4, pp. 1554-1564, 2018.

[36] X. Xu, Y. Liu, H. Li, and F. E. Alsaadi, "Robust set stabilization of Boolean control networks with impulsive effects," Nonlinear Analysis: Modelling and Control, vol. 23, no. 4, pp. 553-567, 2018.

[37] X. Xu, Y. Liu, H. Li, and F. E. Alsaadi, "Synchronization of switched Boolean networks with impulsive effects," International Journal of Biomathematics, vol. 11, no. 6, Article ID 1850080, 2018.

[38] X. Zhang, X. Li, and X. Han, "Design of hybrid controller for synchronization control of Chen chaotic system," The Journal of Nonlinear Sciences and Applications, vol. 10, no. 6, pp. 3320-3327, 2017.

[39] G. Zhao, Y. Wang, and H. Li, "A matrix approach to modeling and optimization for dynamic games with random entrance," Applied Mathematics and Computation, vol. 290, pp. 9-20, 2016.

[40] Z. Zhou and N. N. Yan, "A survey of numerical methods for convection-diffusion optimal control problems," Journal of Numerical Mathematics, vol. 22, no. 1, pp. 61-85, 2014.

[41] S. Ji, G. Li, and M. Wang, "Controllability of impulsive differential systems with nonlocal conditions," Applied Mathematics and Computation, vol. 217, no. 16, pp. 6981-6989, 2011.

[42] J. Prüss, "Evolutionary integral equations and applications," in Monographs in Mathematics, vol. 87Basel, Switzerland, Birkhäuser Verlag, 1993.

[43] D. Guo, V. Lakshmikantham, and X. Liu, Nonlinear Integral Equations in Abstract Spaces, Kluwer Academic Publishers, Dordrecht, Netherlands, 1996.

[44] J. Liang and H. Yang, "Controllability of fractional integrodifferential evolution equations with nonlocal conditions," Applied Mathematics and Computation, vol. 254, pp. 20-29, 2015. 